\title{
CRC-Aided Belief Propagation List Decoding of Polar Codes
}

\author{
Marvin Geiselhart, Ahmed Elkelesh, Moustafa Ebada, Sebastian Cammerer and Stephan ten Brink \\ Institute of Telecommunications, Pfaffenwaldring 47, University of Stuttgart, 70569 Stuttgart, Germany \\ \{geiselhart,elkelesh,ebada,cammerer,tenbrink\}@inue.uni-stuttgart.de
}

\begin{abstract}
Although iterative decoding of polar codes has recently made huge progress based on the idea of permuted factor graphs, it still suffers from a non-negligible performance degradation when compared to state-of-the-art CRC-aided successive cancellation list (CA-SCL) decoding. In this work, we show that iterative decoding of polar codes based on the belief propagation list (BPL) algorithm can approach the error-rate performance of CA-SCL decoding and, thus, can be efficiently used for decoding the standardized 5G polar codes. Rather than only utilizing the cyclic redundancy check (CRC) as a stopping condition (i.e., for error-detection), we also aim to benefit from the error-correction capabilities of the outer CRC code. For this, we develop two distinct soft-decision CRC decoding algorithms: a Bahl-Cocke-Jelinek-Raviv (BCJR)-based approach and a sum product algorithm (SPA)-based approach. Further, an optimized selection of permuted factor graphs is analyzed and shown to reduce the decoding complexity significantly. Finally, we benchmark the proposed $\mathrm{CRC}$-aided belief propagation list (CA-BPL) to state-of-the-art 5G polar codes under CA-SCL decoding and, thereby, showcase an error-rate performance not just close to the CA-SCL but also close to the maximum likelihood (ML) bound as estimated by ordered statistic decoding (OSD).
\end{abstract}

\section{INTRODUCTION}

Polar codes [1] are the first type of channel codes which are theoretically proven to achieve channel capacity. However, this is only true when the code length tends to infinity under a low complexity successive cancellation (SC) decoder. Due to the recent advances in polar code design and polar decoding, short length cyclic redundancy check (CRC)-aided polar codes are selected as the channel code for the uplink and downlink control channel of the upcoming 5G standard [2]. The concatenation of an outer CRC code is important to enchance the short-length error-correcting capabilities of the polar code. Thus, efficiently decoding short CRC-aided polar codes in terms of error-rate performance and decoding complexity/latency is an active topic of research, in particular for ultra-reliable low-latency communications (URLLC).

The state-of-the-art successive cancellation list (SCL) decoder of polar codes [3] benefits significantly from the possibility of a seamless integration of the CRC into the decoding process. For this, the CRC is used to pick the correct candidate from the list of possible codewords. However, the SCL algorithm suffers from unfavorable decoding latency due to its sequential decoding nature and is also not well suited for iterative detection and decoding due to the hardoutput nature of the decoder (and its latency). Another polar decoding approach is to use an iterative belief propagation

This work has been supported by DFG, Germany, under grant BR 3205/5-1.
(BP) decoder [4] which is inherently parallel and, thus, allows high throughput implementations [5] [6]. Also, it is a softin/soft-out (SISO) decoder and, thus, is very suitable for iterative detection and decoding. However, its main drawback is a degraded error-rate performance when compared to the CRC-aided successive cancellation list (CA-SCL) decoder.

Several algorithms have been proposed over the past few years to enhance the error-rate performance of iterative polar decoding algorithms. Among these was an outer graph-based code (e.g., low-density parity-check (LDPC) code [7] or polar code [8]) which was (partially) augmented to the inner polar code factor graph to enhance the reliability of the semipolarized bit-channels. In [9], [10], virtual noise of low power is added to the decoder input (i.e., channel output) to avoid falling into trapping/stopping sets which also enhances the error-rate of the BP decoder. Furthermore, polar codes can be decoded over different permuted factor graphs which can greatly enhance the error-rate performance. This was studied for different channels (e.g., Binary Erasure Channel (BEC) [11] and additive white Gaussian noise (AWGN) [12]) and different decoders (e.g., BP [13] and SCL [14]). It is worth mentioning that similar ideas were also investigated for ReedMuller (RM) codes [14], [15].

Iterative decoding of CRC-aided polar codes was studied in few recent works (e.g., with only CRC detection in [12] and with the aid of the CRC error-correcting capabilities in [16]). However, to the best of our knowledge, there exists no iterative decoding algorithm of polar codes which achieves (or approaches) the performance of the CRC-aided polar codes under SCL decoding (with a sufficiently large list size).

Belief propagation list (BPL) decoding of polar codes [13] is an iterative BP-based decoding in which a list of different BP decoders run in parallel, each with a different polar code factor graph (FG). It was shown that it achieves the same error-rate performance as SCL decoding of plain polar codes (i.e., no outer CRC code). In this work, we focus on solving some open problems in the BPL decoder such as: 1.) CRC incompatibility and 2.) reducing complexity by optimizing the factor graph selection. We combine the idea of iterative decoding of polar codes over permuted factor graphs with SISO decoding of the $\mathrm{CRC}$, utilizing the error-correction capabilities of the CRC (rather than only detection of errors). Our results, surprisingly, show that it is possible to approach the CRC-aided SCL errorrate performance with a BP-based decoding framework. As such, the proposed algorithm suggests a competitive decoding to CA-SCL for CRC-aided polar codes. 


\section{Polar Codes And Iterative Decoding}

Polar codes are based on the channel polarization concept, where $N$ synthesized channels show a polarization behavior (good and bad channels). Practically, polar codes can be seen as a wide range of codes, each characterized by a set of good bit-channels or its complementary set of bad channels (denoted as the information set $\mathbb{A}$ and the frozen set $\mathbb{A}_{c}$, respectively).

The polarization matrix of polar codes of block size $N=2^{n}$ is given by $\mathbf{G}_{N}=\mathbf{F}^{\otimes n}$, where $\mathbf{F}=\left[\begin{array}{ll}1 & 0 \\ 1 & 1\end{array}\right]$ and $\mathbf{F}^{\otimes n}$ denotes the $n$-th Kronecker power of $\mathbf{F}$. The $k \times N$ polar code generator matrix $\mathbf{G}$ is a sub-matrix of $\mathbf{G}_{N}$ with row indices corresponding to $\mathbb{A} .{ }^{1}$ Throughout this work, we use the notation $\mathscr{P}(N, k)$ to denote a polar code of length $N$ and code dimension $k$.

\section{A. Belief Propagation Decoding}

Unlike the SC-based polar decoders, an iterative BP decoding scheme [4] is based on the idea of message passing over the encoding FG, shown in Fig. 3 (rightmost box). Finally, a hard decision is applied on the resultant log-likelihood ratio (LLR) values on the left or the right of the FG in order to recover the information bits (in the vector $\hat{\mathbf{u}}$ ) or the transmitted codeword $\hat{\mathbf{x}}$, respectively.

The conventional BP decoder, $\mathrm{BP}\left(N_{\mathrm{it}, \max }\right)$, terminates when a maximum number of iterations $N_{\mathrm{it}, \max }$ is reached. Early stopping conditions can speed up the decoding process [17]:

- G-based: decoding terminates when $\hat{\mathbf{x}}=\hat{\mathbf{u}} \cdot \mathbf{G}_{N}$.

- CRC-based: decoding terminates when the checks of the concatenated CRC code are satisfied. It is important to keep in mind that in this case, the CRC code only acts as a stopping condition (i.e., error-detection).

\section{B. Belief Propagation List (BPL) Decoding}

It has been observed that the stages of the encoding graph of a polar code of length $2^{n}$ can be permuted leading to $n$ ! different graphs with the same encoding behavior [11]. This enables the possibility of BP decoding on different realizations of the FG. Seeing that different permutations contain different loops, one FG permutation may be better than another permutation for a specific input (i.e., transmitted codeword plus noise realization). BPL exploits this idea by using a set $\mathbb{S}$ of $L$ parallel independent BP decoders each with a different permutation. Iterative decoding is conducted on the $L$ decoders in parallel with $N_{\mathrm{it}, \max }$ iterations per decoder and only those codewords satisfying the G-matrix-based stopping condition are declared as the set of valid polar codewords. Finally, that valid codeword from the $L$ parallel BP decoders which is closest, in terms of Euclidean distance, to the channel output $\mathbf{y}$ is picked to be the BPL decoder output. Throughout this work, we use the notation BPL $\left(L, N_{\mathrm{it}, \max }\right)$ to denote this decoder. For more details, we refer the interested reader to [13].

Interestingly, it was shown in [18] that a stage-wise permutation on the FG corresponds to certain bit-wise permutations

\footnotetext{
${ }^{1}$ Based on this insight, RM codes can be easily related to polar codes [11].
}

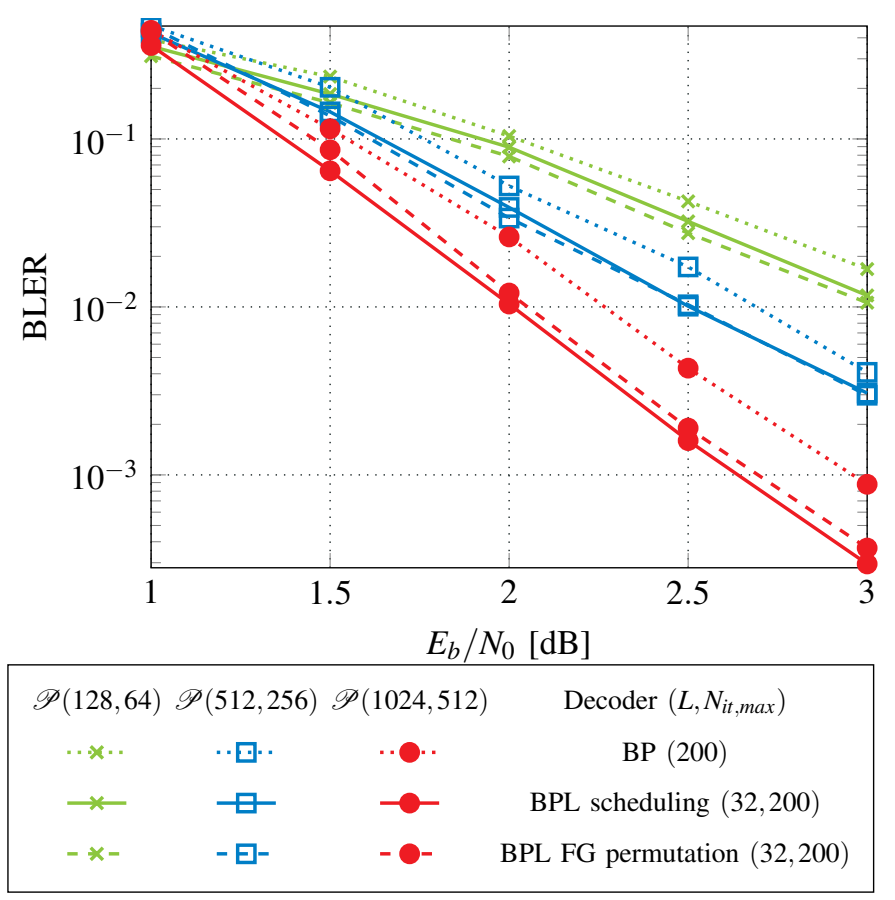

Fig. 1: BLER comparison between BPL with permuted scheduling and permuted factor graphs (FGs); $5 \mathrm{G}$ polar codes of rate 0.5 (i.e., $\mathscr{P}(N, N / 2)$ codes); no CRC is used.

of the vectors $\mathbf{u}$ and $\mathbf{x}$. This enables permuted-decoding on the same FG while only doing these bit-mappings. It also enabled the same permuted-decoder concept with other decoders without having to change the decoder structure itself which is highly useful for hardware implementations (see [14]).

The selection of permutation sets $\mathbb{S}$ was originally done in a random manner [12]. Later, it was shown that a smart permutations selection would introduce additional performance gains [18]-[21]. However, an optimal FG selection strategy remains an open problem.

\section{Factor Graph Permutations vs. Decoding Schedule Permu- tations}

Two implementation strategies have been found to realize the BPL decoding of polar codes:

1) Graph permutations, as originally introduced in [13] where the FG stages are permuted according to a specific permutation. Afterwards, conventional BP decoding is conducted on the permuted factor graph, following the conventional decoding schedule (i.e., permuted stages from 1 to $n$, or $n$ to 1 , are updated sequentially).

2) Decoding schedule permutations, where conventional $B P$ decoding is conducted on the conventional factor graph, while permuting the decoding schedule (i.e., permuting the order in which stages are being activated/updated).

Fig. 1 depicts that both strategies have a similar errorrate performance, for different code lengths. We empirically observed that the factor graph permutation-based BPL yields a better performance in the short block length regime, while its scheduling-based counterpart performs better for longer code lengths. 

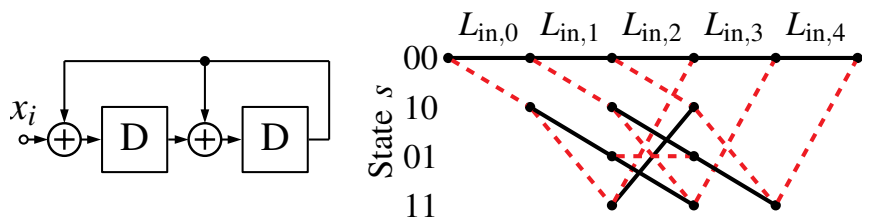

Fig. 2: Linear feedback shift register (LFSR) and length $n_{\mathrm{CRC}}=5$ trellis of the CRC code with $g(x)=x^{2}+x+1$.

\section{CRC-AIDED BPL DECODING}

None of the previously described BPL decoding schemes utilizes the presence of an outer CRC code during decoding. In this paper, we propose a CRC-aided belief propagation list (CA-BPL) decoding scheme that extends the standard BPL decoding. Its underlying idea is to utilize estimated softinformation from the CRC code to further enhance the BPL decoder (i.e., we treat the CRC as an outer code component). Accordingly, we present two SISO decoding algorithms for the CRC code that enhance the error-correcting capability of the BPL decoder.

CRC codewords are defined as polynomials of degree less than $n_{\mathrm{CRC}}$ that are divisible by the generator polynomial $g(x)$ of degree $r$. To check divisibility, a linear feedback shift register (LFSR) of length $r$ with input $x_{i}$ and feedback according to $g(x)$ can be used. The CRC code can be visualized in a trellis diagram with the state $s$ of the shift register on the vertical axis and the discrete time index $i$ on the horizontal axis. Fig. 2 shows the LFSR and the trellis of a CRC-2 code with polynomial $g(x)=x^{2}+x+1$ as an example. The register is initialized to the all-zero state. Every time index, a state transition $\left(s^{\prime}, s\right)$ from state $s^{\prime}$ to $s$ occurs, depending on the input bit value $x_{i}$. A bit sequence fulfills the CRC, i.e., it is a codeword, if and only if the shift register returns to the all-zero state when the last bit enters the circuit. For all transitions, $\lambda_{\text {trans }}\left(s^{\prime}, s\right) \in\{0,1\}$ denotes the bit value of the transition. Likewise, $U_{0}$ and $U_{1}$, depicted as solid black and dashed red lines in the trellis diagram, denote the set of edges $\left(s^{\prime}, s\right)$ corresponding to the 0 and 1 transitions, respectively.

We use the Bahl-Cocke-Jelinek-Raviv (BCJR) algorithm [22] to enable maximum a posteriori (MAP) decoding of the CRC code. Thus, we briefly present the log-domain BCJR algorithm [23].

$$
\tilde{\alpha}_{i+1}(s)=\max _{s^{\prime}}{ }^{*}\left(\tilde{\alpha}_{i}\left(s^{\prime}\right)-L_{\mathrm{in}, i} \cdot \lambda_{\text {trans }}\left(s^{\prime}, s\right)\right)
$$

$\forall s$ and $i=0, \ldots, n_{\mathrm{CRC}}-1$ starting with $\tilde{\alpha}_{0}(0)=0$.

$$
\tilde{\beta}_{i}\left(s^{\prime}\right)=\max _{s} *\left(\tilde{\beta}_{i+1}(s)-L_{\mathrm{in}, i} \cdot \lambda_{\text {trans }}\left(s^{\prime}, s\right)\right)
$$

$\forall s^{\prime}$ and $i=n_{\mathrm{CRC}}-1, \ldots, 0$ starting with $\tilde{\beta}_{n_{\mathrm{CRC}}}(0)=0$.

$$
L_{\text {out }, i}=\max _{U_{0}}^{*}\left(\tilde{\alpha}_{i}\left(s^{\prime}\right)+\tilde{\beta}_{i+1}(s)\right)-\max _{U_{1}}^{*}\left(\tilde{\alpha}_{i}\left(s^{\prime}\right)+\tilde{\beta}_{i+1}(s)\right) \text {. }
$$

The inputs to the BCJR algorithm are the LLRs $L_{\mathrm{in}, i}$ of each bit of the noisy CRC codeword. The BCJR algorithm estimates a posteriori $\log$-probabilities $\tilde{\alpha}$ and $\tilde{\beta}$ recursively, based on the current state along with state transition probabilities in a forward pass (1) and a backward pass (2) on the trellis. The
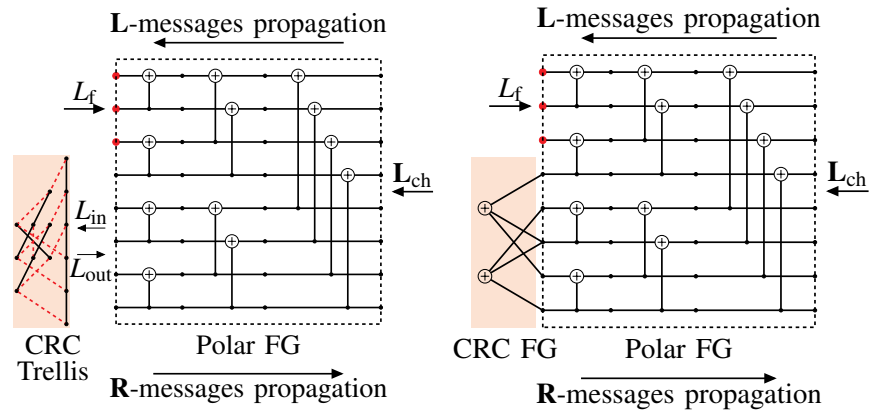

Fig. 3: Polar + CRC decoders based on the polar factor graph (FG) augmented with BCJR trellis (left) and SPA factor graph (right) for the CRC. The code used is a $\mathscr{P}(8,5)$ polar code concatenated with a CRC code with polynomial $g(x)=x^{2}+x+1$.

estimates are combined in a final step (3) to obtain the extrinsic L-value $L_{\text {out }, i}$ for each bit. To compute the sum of probabilities in the log-domain, we use the max* operator, which can be expressed as $\max _{j}^{*}\left(x_{j}\right)=\log \left(\sum_{j} e^{x_{j}}\right)$.

From the equations it is obvious that the complexity of the BCJR algorithm scales proportional to the number of states in the underlying state machine. Therefore, the overall complexity is $\mathscr{O}\left(n_{\mathrm{CRC}} \cdot 2^{r}\right)$, making this algorithm infeasible for long CRC lengths $r$.

To counteract complexity, we propose a second SISO decoder. It is based on the sum product algorithm (SPA) used to decode LDPC codes. SPA operates on the parity-check matrix $\mathbf{H}_{\mathrm{CRC}}$ of the CRC code. For details on the SPA, we refer the interested reader to [23]. $\mathbf{H}_{\mathrm{CRC}}$ is directly obtained from the systematic generator matrix, which is generated by the CRC codewords corresponding to the unit vectors. As the SPA is known to have poor performance on high density parity-check matrices, the density of $\mathbf{H}_{\mathrm{CRC}}$ is reduced using row operations. As a heuristic, a greedy algorithm that iteratively combines two rows and replaces the one with higher Hamming weight, proved to be sufficient for this task. ${ }^{2}$

For the example CRC code with polynomial $g(x)=x^{2}+x+1$, the resulting parity-check matrix is

$$
\mathbf{H}_{\mathrm{CRC}}=\left[\begin{array}{lllll}
1 & 0 & 1 & 1 & 0 \\
0 & 1 & 1 & 0 & 1
\end{array}\right] .
$$

Fig. 3 shows how both the BCJR and SPA decoders of the CRC code are augmented to the left side of the polar decoder. The CRC decoder only interacts with the non-frozen bit-positions $\mathbb{A}$ of the polar code. After each right-to-left message propagation on the polar FG, the $\mathrm{CRC}$ decoder is fed with the left-most L-messages of the information bit channels $\mathbf{L}_{\text {in }}=\mathbf{L}_{0, \mathbb{A}}$. Then, the CRC decoder is updated and its output is fed back into the left-most R-message $\mathbf{R}_{0, \mathbb{A}}=\mathbf{L}_{\text {out }}$, followed by a left-to-right message update on the polar FG.

In CA-BPL, a total of $L$ permuted factor graphs is used, each with its own instance of the CRC decoder. Throughout this work, we only consider factor graph permutation-based BPL rather than scheduling-based BPL (cf. Fig. 1).

\footnotetext{
${ }^{2}$ We empirically observed that the exact form of $\mathbf{H}_{\mathrm{CRC}}$ has only minor impact on the error-rate performance.
} 


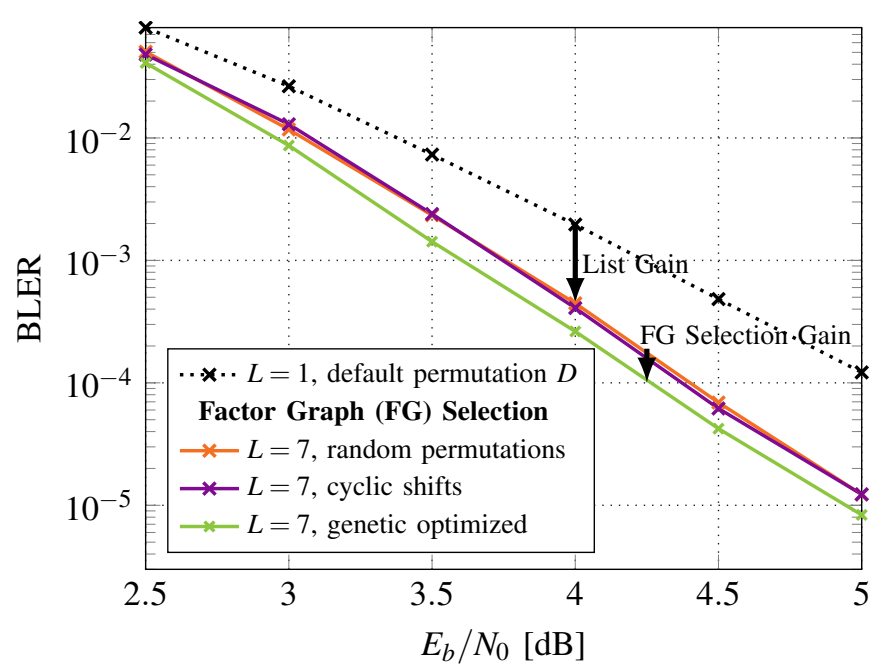

Fig. 4: BLER comparison between CRC-aided BPL with different permutation selection methods; BCJR-based CRC decoders were used and $N_{\text {it,max }}=200$. The considered CRC-aided polar code has a code length $N=128$, code dimension $k=64$ and CRC- 6 is used, thus, the overall code rate is 0.5 .

\section{RESULTS}

We evaluate the performance of the CA-BPL algorithm on a concatenated polar-CRC code with codelength $N=128$ and code dimension $k=64$. As CRC polynomial, CRC-6 from the $5 \mathrm{G}$ standard [2] $g(x)=x^{6}+x^{5}+1$ is used. Consequently, the polar code has 70 non-frozen bits, to account for the rate loss of the outer CRC code. For the sake of reproducibility, we use the bit-reliability order specified in the $5 \mathrm{G}$ standard [2] as the design criterion of the inner polar code (i.e., $\mathbb{A}$ ).

\section{A. Permutation Selection}

One possible approach to reduce the complexity of the proposed decoding scheme would be to smartly select the FG permutations reducing the number of required FGs.

Remember that, in BPL, as long as the received vector is maximum likelihood (ML)-decodable, only one of the $L$ permuted BP decoders has to converge to the correct codeword to successfully decode. In other words, the block error probability of the ML-decodable received sequences $P\left(E_{\mathrm{BPL}} \mid \overline{E_{\mathrm{ML}}}\right)$ of the BPL decoder is the probability of all the constituent BP decoders being in error. This is expressed as

$$
P\left(E_{\mathrm{BPL}(\mathbb{S})} \mid \overline{E_{\mathrm{ML}}}\right)=P\left(\bigcap_{i \in \mathbb{S}} E_{i} \mid \overline{E_{\mathrm{ML}}}\right)
$$

where $\overline{E_{\mathrm{ML}}}$ denotes an ML decoder success event, $E_{\mathrm{BPL}}$ denotes the event of the BPL decoder block error, and $E_{i}$ denotes the event of the BP decoder using FG permutation $i$ not converging to the correct codeword within $N_{\text {it,max }}$ iterations.

Therefore, to use the full potential of BPL, it is necessary to find a set $\mathbb{S}$ of $L$ factor graph permutations that yields the best joint block error rate (BLER) performance. The intuition behind this idea is to have more diverse permutations that lead to more differentiated results (i.e., diversity in the final list of candidates) than multiple good permutations that lead to the same results (i.e., having the same candidate multiple times in the list). Thus, we would like to minimize $P\left(E_{\mathrm{BPL}(\mathbb{S})} \mid \overline{E_{\mathrm{ML}}}\right)$ over all possible sets $\mathbb{S}$ with the constraint $|\mathbb{S}|=L$

$$
\begin{array}{ll}
\underset{\mathbb{S}}{\operatorname{minimize}} & P\left(\bigcap_{i \in \mathbb{S}} E_{i} \mid \overline{E_{\mathrm{ML}}}\right) \\
\text { subject to } & |\mathbb{S}|=L .
\end{array}
$$

For a length $N=128$ code, there exist $7 !=5040$ different permutations of the FG. Thus, the search space of the optimization problem contains $\left(\begin{array}{c}5040 \\ L\end{array}\right)$ possible permutation sets. As we can only estimate the probabilities using Monte Carlo simulation and error events are comparably rare, naive optimization is computationally infeasible. For the $5 \mathrm{G}$ construction of the polar code, however, we empirically observe that, regarding standalone BP decoders, the default permutation $D$ achieves the lowest BLER. By assuming $D$ is in the optimum set $\mathbb{S}$ we can factor equation (4) into a conditional BP BLER and a term corresponding to the list gain:

$$
P\left(\bigcap_{i \in \mathbb{S}} E_{i} \mid \overline{E_{\mathrm{ML}}}\right) \stackrel{D \in \mathbb{S}}{=} P\left(E_{D} \mid \overline{E_{\mathrm{ML}}}\right) \cdot \underbrace{P\left(\bigcap_{i \in \mathbb{S} \backslash\{D\}} E_{i} \mid E_{D}, \overline{E_{\mathrm{ML}}}\right)}_{\text {List Gain }} .
$$

This factorization simplifies the optimization greatly, as $P\left(E_{i} \mid E_{D}, \overline{E_{\mathrm{ML}}}\right) \gg P\left(E_{i} \mid \overline{E_{\mathrm{ML}}}\right)$, due to the correlation of the permuted FG decoders. Consequently, a comparably small dataset of $2 \cdot 10^{4}$ samples could be used to find the remaining optimal $L-1$ other permutations. The collected dataset contains $\mathbf{y}$ vectors at $E_{\mathrm{b}} / N_{0}=4 \mathrm{~dB}$ for which the BP decoder, using the default permutation $D$, fails to decode. We then evaluate the convergence of all other 5039 permutations. Finally, a genetic algorithm is applied on 15000 samples of the dataset to find the optimal $\mathbb{S}$ by minimizing the estimate of $P\left(\bigcap_{i \in \mathbb{S} \backslash\{D\}} E_{i} \mid E_{D}\right)$, i.e., maximizing the list gain. The last 5000 samples serve as a validation set to test whether the solution generalizes well. In Fig. 4 we compare different permutation selection methods for CA-BPL with list size $L=7$ based on BLER performance. The genetic optimized list outperforms both random permutations and the 7 cyclic shifts of the default permutation.

\section{B. Error-Rate Performance}

We compare a classical BPL decoder that uses the CRC solely as a stopping condition to our proposed CA-BPL decoding scheme, with both BCJR and SPA CRC decoders. Additionally, we use CA-SCL decoding with list size $L=32$ and order-4 ordered statistic decoding (OSD) [24] as a lower bound on the BLER performance. The CRC parity-check ensemble used has (edge-perspective) variable node and check node degree distribution polynomials $\lambda(Z)=0.06+0.16 Z^{1}+0.3 Z^{2}+0.3 Z^{3}+0.15 Z^{4}+0.03 Z^{5}$ and $\rho(Z)=0.66 Z^{32}+0.34 Z^{33}$, respectively.

The BPL and CA-BPL decoders use $N_{\text {it,max }}=200$ iterations and $L=32$ parallel decoders. The FG permutations were selected using the optimization technique described above. Fig. 5 shows the BLER performance of the decoders over the AWGN channel using binary phase shift keying (BPSK) modulation. The proposed CA-BPL scheme outperforms a classical BPL (with $\mathrm{CRC}$ used as a stopping criterion) by 


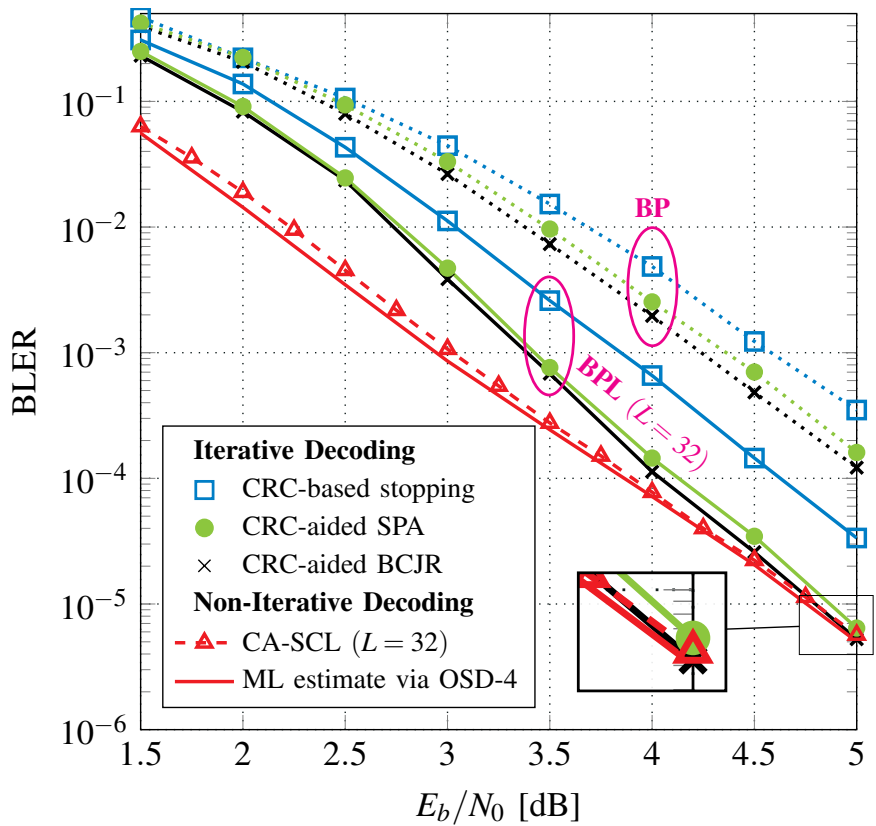

Fig. 5: BLER comparison between different CRC-aided BPL decoders and CRC-aided SCL decoder; the considered CRC-aided polar code has a code length $N=128$, code dimension $k=64$ and CRC- 6 is used, thus, the overall code rate is 0.5 . The iterative decoders use a maximum number of iterations of $N_{\text {it,max }}=200$.

$0.5 \mathrm{~dB}$ at a BLER of $10^{-3}$. Note that, this gain is solely due to benefiting from the CRC error-correction capabilities rather than only detecting errors. It is worth mentioning that CA-BPL approaches the performance of CA-SCL closer than $0.15 \mathrm{~dB}$ at a BLER of $10^{-4}$. The same BLER performance as CA-SCL is reached at $E_{\mathrm{b}} / N_{0}=5 \mathrm{~dB}$. It is quite remarkable that the much simpler SPA-aided BPL decoder is only marginally worse than the (optimal) BCJR-aided BPL.

\section{CONCLUSION}

We have introduced a CRC-aided extension of the iterative BPL decoding algorithm, where the CRC is used for errorcorrection rather than the plain error-detection. For this, we have developed two soft-in/soft-out decoding techniques for CRC codes based on the trellis-based BCJR algorithm and the iterative SPA decoder. To account for the additional decoding overhead, we have further optimized the selection of factor graph permutations and have shown that the required list size can be reduced. As a result, the proposed CA-BPL algorithm can compete with CA-SCL decoding in the typical SNR region of interest while promising lower decoding latency and offering the potential for parallel algorithm implementations. For a large enough list size, the proposed algorithm even approaches the estimated ML performance of the concatenated polar+CRC coding scheme. To the best of our knowledge, these are the best iterative polar+CRC decoding results that have been reported in literature. Although we have given some intuition why the selection of factor graph permutation works, we leave it open for future work to derive a theoretical analysis of why a certain set of permutations yields a better overall performance than other selections.

\section{REFERENCES}

[1] E. Arıkan, "Channel Polarization: A Method for Constructing CapacityAchieving Codes for Symmetric Binary-Input Memoryless Channels," IEEE Trans. Inf. Theory, vol. 55, no. 7, pp. 3051-3073, Jul. 2009.

[2] "Technical Specification Group Radio Access Network," 3GPP, 2018 TS 38.212 V.15.1.1. [Online]. Available: http://www.3gpp.org/ftp/Specs/ archive/38_series/38.212/

[3] I. Tal and A. Vardy, "List Decoding of Polar Codes," IEEE Trans. Inf. Theory, vol. 61, no. 5, pp. 2213-2226, May 2015.

[4] E. Arikan, "Polar Codes: A Pipelined Implementation," Proc. 4th ISBC, pp. 11-14, 2010.

[5] S. Sun and Z. Zhang, "Architecture and Optimization of HighThroughput Belief Propagation Decoding of Polar Codes," in IEEE Inter. Symp. on Circuits Syst. (ISCAS), May 2016, pp. 165-168.

[6] S. Cammerer, B. Leible, M. Stahl, J. Hoydis, and S. ten Brink, "Combining Belief Propagation and Successive Cancellation List Decoding of Polar Codes on a GPU Platform," in IEEE Inter. Conf. on Acoustics, Speech, and Sig. Process. (ICASSP), Mar. 2017, pp. 3664-3668.

[7] J. Guo, M. Qin, A. G. i Fàbregas, and P. H. Siegel, "Enhanced Belief Propagation Decoding of Polar Codes through Concatenation," in IEEE Inter. Symp. Inf. Theory (ISIT), Jun. 2014, pp. 2987-2991.

[8] A. Elkelesh, M. Ebada, S. Cammerer, and S. ten Brink, "Flexible Length Polar Codes through Graph Based Augmentation," in IEEE Inter. ITG Conf. on Syst., Commun. and Coding (SCC), Feb. 2017.

[9] A. Elkelesh, S. Cammerer, M. Ebada, and S. ten Brink, "Mitigating Clipping Effects on Error Floors under Belief Propagation Decoding of Polar Codes," in Inter. Symp. Wireless Commun. Syst., Aug. 2017.

[10] A. Çağrı Arlı and O. Gazi, "Noise-Aided Belief Propagation List Decoding of Polar Codes," IEEE Commun. Lett., vol. 23, no. 8, pp. 1285-1288, Aug. 2019.

[11] N. Hussami, S. B. Korada, and R. Urbanke, "Performance of Polar Codes for Channel and Source Coding," in IEEE Inter. Symp. Inf. Theory (ISIT), Jun. 2009, pp. 1488-1492.

[12] A. Elkelesh, M. Ebada, S. Cammerer, and S. ten Brink, "Belief Propagation Decoding of Polar Codes on Permuted Factor Graphs," in IEEE Wireless Commun. and Networking Conf. (WCNC), Apr. 2018.

[13] — , "Belief Propagation List Decoding of Polar Codes," IEEE Commun. Lett., vol. 22, no. 8, pp. 1536-1539, Aug. 2018.

[14] S. A. Hashemi, N. Doan, M. Mondelli, and W. J. Gross, "Decoding Reed-Muller and Polar Codes by Successive Factor Graph Permutations," in IEEE 10th Inter. Symp. on Turbo Codes Iterative Inf. Process. (ISTC), Dec. 2018.

[15] K. Ivanov and R. Urbanke, "Permutation-based Decoding of ReedMuller Codes in Binary Erasure Channel," in IEEE Inter. Symp. Inf. Theory (ISIT), Jul. 2019.

[16] N. Doan, S. A. Hashemi, E. N. Mambou, T. Tonnellier, and W. J. Gross, "Neural Belief Propagation Decoding of CRC-Polar Concatenated Codes," in IEEE Inter. Conf. on Commun. (ICC), May 2019.

[17] B. Yuan and K. K. Parhi, "Early Stopping Criteria for Energy-Efficient Low-Latency Belief-Propagation Polar Code Decoders," IEEE Trans. Sig. Process., vol. 62, no. 24, pp. 6496-6506, Dec. 2014.

[18] N. Doan, S. A. Hashemi, M. Mondelli, and W. J. Gross, "On the Decoding of Polar Codes on Permuted Factor Graphs," in IEEE Global Commun. Conf. (GLOBECOM), Dec. 2018.

[19] Z. Q. Chen, L. Li, Z. Ma, and P. Z. Fan, "List Selection and Decision Fusion Scheme for Belief Propagation List Decoding of Polar Codes," in IEEE 30th Annual Inter. Symp. on Personal, Indoor and Mobile Radio Commun. (PIMRC), Sep. 2019.

[20] V. Ranasinghe, N. Rajatheva, and M. Latva-aho, "Partially Permuted Multi-Trellis Belief Propagation for Polar Codes," ArXiv e-prints, Nov. 2019.

[21] B. Tosun, "Belief Propagation Decoding Using Factor Graph Permutations," Master thesis, Middle East Technical University, 2019.

[22] L. Bahl, J. Cocke, F. Jelinek, and J. Raviv, "Optimal Decoding of Linear Codes for Minimizing Symbol Error Rate," IEEE Trans. Inf. Theory, vol. 20, no. 2, pp. 284-287, Mar. 1974.

[23] W. E. Ryan and S. Lin, "Channel Codes: Classical and Modern," Cambridge University Press, 2009.

[24] M. Fossorier and S. Lin, "Soft-Decision Decoding of Linear Block Codes Based on Ordered Statistics," IEEE Trans. Inf. Theory, vol. 41, no. 5, pp. 1379-1396, Sep. 1995. 